\title{
Anatomical vs Anti-anatomical Placement of a Tilting-disk Mechanical Valve in Mitral Position
}

${ }^{1}$ GN Chennakeshavallu, ${ }^{2}$ Shrinivas Gadhinglajkar, ${ }^{3}$ Rupa Sreedhar, ${ }^{4}$ Prashanth P Bhaskar, ${ }^{5}$ Varghese T Panicker

\begin{abstract}
Anatomical and anti-anatomical placement of a prosthetic mechanical valve in mitral position has been described in the literature. In mitral position, it is preferred to place the tiltingdisk valve with its large orifice directed posteriorly, which is called as "anatomical position." We report an incident wherein a Chitra heart valve prosthesis (CHVP) implanted in anatomical position had limited motion of tilting disk with inadequate angle of opening. This was addressed by rotating the prosthesis occluder mechanism by $180^{\circ}$ in situ in such a way that the large orifice was directed anteriorly (anti-anatomical position) and this resulted in the adequate opening of the tilting disk. Transesophageal echocardiography (TEE) played a decisive role in identifying the problem and ensuring adequacy of disk opening after repositioning the prosthesis.
\end{abstract}

Keywords: Anatomical position, Anti-anatomical position, Chitra heart valve prosthesis, Mitral valve prosthesis, Transesophageal echocardiography.

How to cite this article: Chennakeshavallu GN, Gadhinglajkar S, Sreedhar R, Bhaskar PP, Panicker VT. Anatomical vs Antianatomical Placement of a Tilting-disk Mechanical Valve in Mitral Position. J Perioper Echocardiogr 2017;5(1):12-15.

Source of support: Nil

Conflict of interest: None

\section{INTRODUCTION}

Prosthetic valve replacement of a diseased native heart valve is indicated in patients with severe valvular heart disease associated with hemodynamically unfavorable conditions. Transesophageal echocardiography is a category I indication for intraoperative assessment of prosthetic valve function. ${ }^{1}$ For optimum hemodynamic performance, in addition to selection of an appropriate

\footnotetext{
${ }^{1,4}$ Senior Resident, ${ }^{2,3}$ Professor, ${ }^{5}$ Associate Professor

${ }^{1-4}$ Department of Cardiovascular and Thoracic Anesthesia, Sree Chitra Tirunal Institute for Medical Sciences and Technology Thiruvananthapuram, Kerala, India

${ }^{5}$ Department of Cardiac Surgery, Sree Chitra Tirunal Institute for Medical Sciences and Technology, Thiruvananthapuram Kerala, India

Corresponding Author: GN Chennakeshavallu, Senior Resident Department of Cardiovascular and Thoracic Anesthesia, Sree Chitra Tirunal Institute for Medical Sciences and Technology Thiruvananthapuram, Kerala, India, Phone: +919914207949 e-mail: chenna.31187@gmail.com
}

make and size of the mechanical prosthesis, importance should be given to the orientation of the occluder mechanism. In order to achieve physiological flow patterns at the mitral position, the bileaflet valves should be implanted with prosthetic leaflet commissure perpendicular to the native valve commissure (anti-anatomical position), whereas the tilting-disk valves should have their larger orifice oriented posteriorly (anatomical position). ${ }^{2,3} \mathrm{We}$ describe a case scenario wherein following placement of a CHVP in mitral position and orienting the larger orifice in anatomical position, we discovered on TEE examination a significant limitation in motion of the disk that resulted in the disk opening at an inadequate angle. Rotating the occluder mechanism by $180^{\circ}$, resulting in the anti-anatomical position of the valve, could restore the normal valve function and adequate opening of the disk. The TEE had a vital role in detecting limitation of leaflet mobility, decision making for the surgical revision, and guiding the surgeon for proper repositioning of the prosthesis.

\section{CASE REPORT}

A 64-year-old diabetic male presented with complaints of dyspnea on exertion (New York Heart Association Class III) and palpitation since 6 months. Preoperative transthoracic echocardiography showed severe mitral stenosis, moderate aortic stenosis, severe aortic regurgitation, and good left ventricular function. Intraoperative TEE (Philips iE33 with X7-2t probe) examination before cardiopulmonary bypass (CPB) revealed thickened, calcified mitral valve leaflets with restricted mobility and severe mitral stenosis (Video 1). Aortic valve assessment revealed thickened calcified leaflets with moderate aortic stenosis and severe aortic regurgitation (Video 2). The aortic annulus was $24 \mathrm{~mm}$ in size. Double valve replacement was performed under moderate hypothermic $\left(32^{\circ} \mathrm{C}\right)$ $\mathrm{CPB}$ and cardioplegic arrest. Both native valves were excised and their annuli were subjected to decalcification to obtain a homogeneous circular surface. Careful inspection of left ventricular cavity showed severely thickened and calcific chordae. Thickened chordae were transected at the junction with papillary muscles. Mitral valve and aortic valve were replaced with 27 and $23 \mathrm{~mm}$ tiltingdisk CHVP respectively. The mitral valve prosthesis was 
implanted directing the larger orifice posteriorly. After rewarming was achieved, TEE was performed while patient was off CPB with aortic and venous cannula in situ. Two-dimensional echocardiography and color Doppler evaluation in different views showed limitation of mitral-disk motion and inadequate opening angle. The mean gradient across mitral valve was $8 \mathrm{mmHg}$. The TEE examination also revealed undue prominence of the aortomitral fibrous region (Fig. 1, Video 3), which was interfering with free movement of anterior aspect of the disk restricting its angle of opening to less than $40^{\circ}$. The decision was made to reposition the valve. The patient was again cooled and cardioplegic arrest reachieved. The prosthesis movements were tested by the surgeon via left atriotomy. This also showed restricted disk opening. The prosthesis was rotated through $180^{\circ}$ in situ with a rotator so as to orient the larger orifice of the valve to open toward the aorta. After weaning the patient from CPB with an inotropic infusion of dobutamine $5 \mu \mathrm{g} / \mathrm{kg} / \mathrm{min}$ and norepinephrine $0.05 \mu \mathrm{g} / \mathrm{kg} / \mathrm{min}$, the cardiac activity of heart was inspected with TEE. It revealed normal mitral prosthetic valve function with disk opening to $70^{\circ}$ (Fig. 2, Video 4) The mean transmitral pressure gradient was $5 \mathrm{~mm} \mathrm{Hg}$. The CHVP implanted in the aortic position was functioning satisfactorily. Patient had an uneventful postoperative course and was discharged from the hospital 7 days later with well-functioning prosthetic valves and mean transmitral Doppler gradient of $3 \mathrm{~mm} \mathrm{Hg}$ as evident on transthoracic echocardiographic examination.

\section{DISCUSSION}

The CHVP is a tilting-disk artificial heart valve designed and developed by Sree Chitra Tirunal Institute for Medical Sciences and Technology. The CHVP consists of an annular metallic stent, which is sutured to the native valve annulus with a sewing ring (Fig. 3). The occluding mechanism is a circular disk, which is suspended from the annular stent by a single strut. The strut attaches the
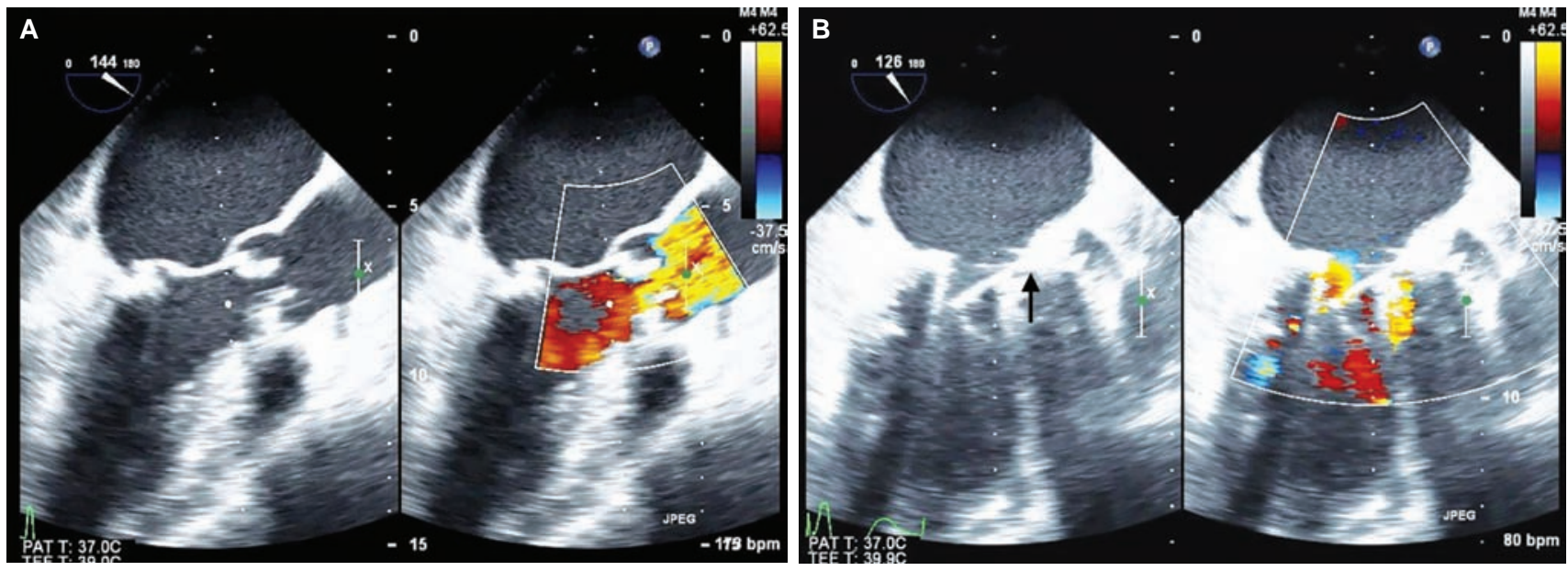

Figs 1A and B: (A) Aorto-mitral region in ME LV long axis view before surgery; and (B) after surgery. Note the prominence of aorto-mitral fibrous region (black arrow) after the surgery which restricts the free opening of the prosthetic disk
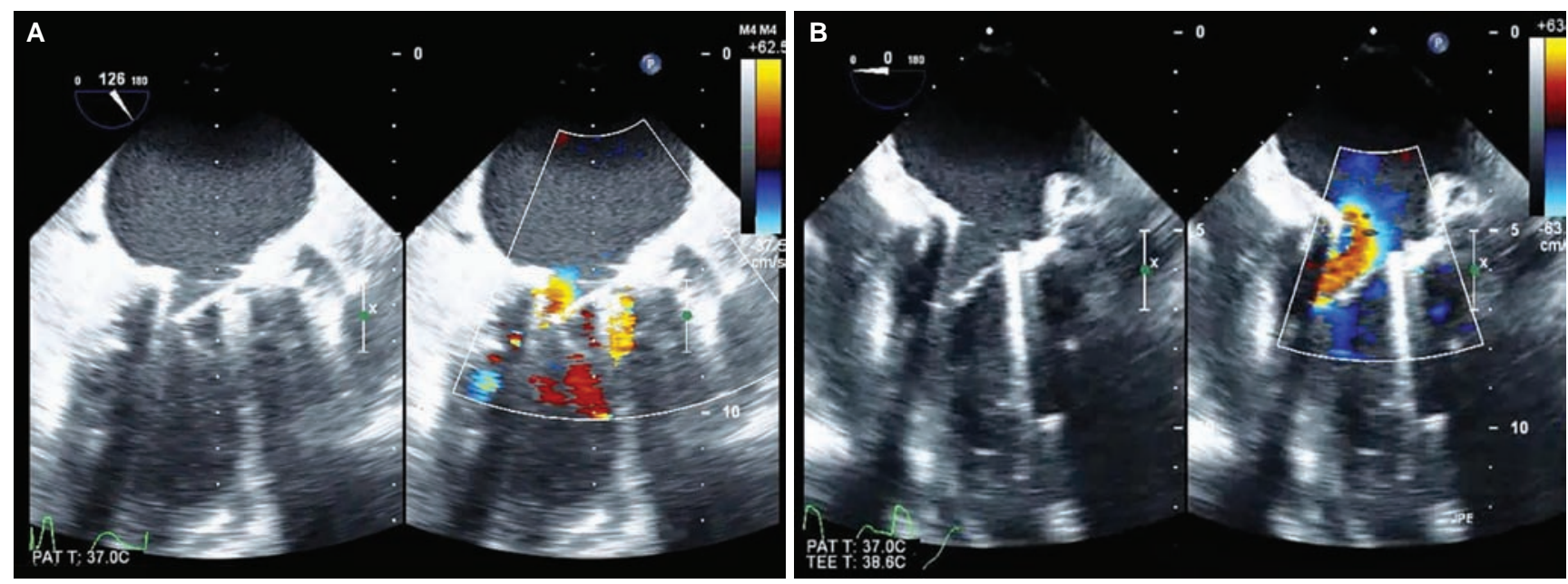

Figs 2A and B: Angle of disk opening has increased after re-positioning the valve [anti-anatomical with large orifice towards aorta as seen in ME 5 chamber view (B)] compared to the opening before re-positioning (anatomical placement with large orifice directed posteriorly away from aorta as seen in ME long axis view (A) 


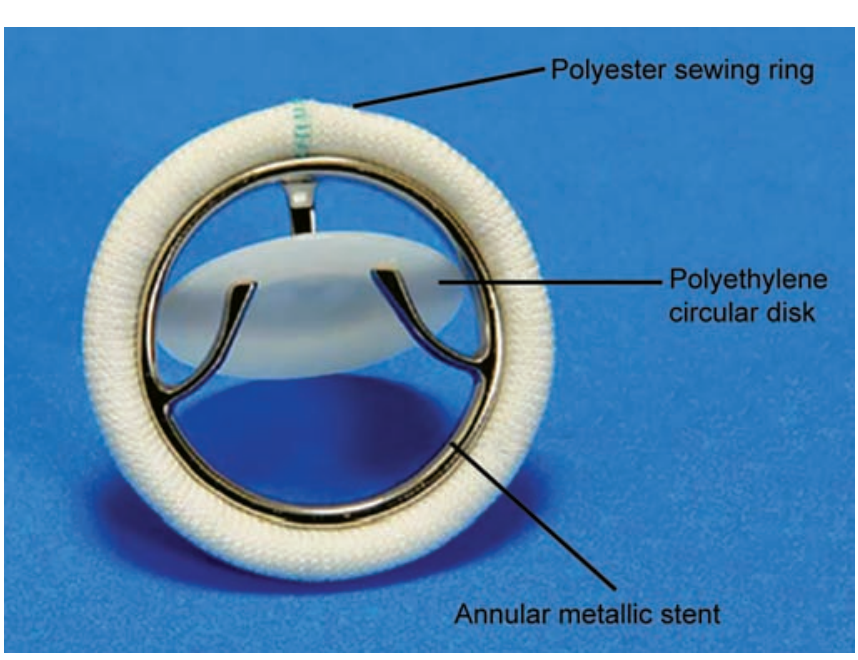

Fig. 3: Structure of Chitra tilting-disk heart valve prosthesis

disk eccentrically so that the backpressure on a larger segment of the disk will tend to close the valve. Eccentric disk position produces two orifices of different sizes and shapes: A major orifice and a minor orifice. The disk occluder pivots open 60 to $75^{\circ}$. The frame and the disk are hydrodynamically designed to reduce drag and inertia and polished to minimize the chances of clotting. It has some attractive features like complete structural integrity, absence of cavitation-related damage, silent operation, rotatable within the sewing ring to assure its freedom to rotate if repositioning is required, and low profile.

Leaflet immobilization following placement of a mechanical prosthesis in mitral position is a relatively rare occurrence. Its incidence and treatment are not well defined. ${ }^{4}$ Obstruction by subvalvular apparatus is the most common mechanism involved in prosthetic leaflet immobilization in the perioperative period. ${ }^{5}$ The subvalvular structures responsible for occluder malfunction may be preserved chordae or inadequately excised native valve leaflets. ${ }^{6}$ Chordal preservation is commonly performed during valve replacement surgery to retain left ventricular geometry. ${ }^{7}$ However, due to thickening and calcification in the rheumatic process, the chordae become less pliable and rigid. Mitral valve leaflets may also become thickened and calcific due to rheumatic process. It may be technically difficult to excise the leaflets completely and surgeon may be left with no option other than to preserve some portion of leaflet along with chordae. Other mechanisms for restricted leaflet mobility of implanted valve are left ventricular myocardium impeding the free movement of prosthetic valve ${ }^{8}$ and immobilization of disk valve by an unraveled suture. ${ }^{9}$ Special care must be taken when implanting prosthetic valves to eliminate the possibility of disk interference caused by suture ends or intracardiac structures. In some cases, the cause of valve malfunction is not determined. Delay in the detection of stuck prosthetic valve results in myocardial distension injury, hemodynamic deterioration, and failure to wean from the CPB. In our case, TEE revealed restricted mobility of the tilting disk at mitral position with an inadequate angle of opening. The undue prominence of the aortomitral fibrous region after replacement of both valves was mechanically hindering free motion of the occluder disk at its anterior portion. The aortomitral fibrous region is frequently involved in the rheumatic process. Replacement of aortic and mitral valve may alter its geometry resulting in a change in its morphology. In our patient, supramitral protrusion of fibrous curtain was interfering with the occluder mechanism. Since surgical intervention to restore the geometry was difficult, rotating the occluder mechanism was the only option left to us. The CHVP has a provision for rotating the occluder mechanism within sewing ring. Rotating the valve in situ by $180^{\circ}$ resulted in major orifice being oriented anteriorly toward the aortic valve. Following this, adequate opening of the tilting disk was restored.

With respect to the surgical implantation of mechanical valves in the mitral position, the surgeon can orient bileaflet valve in such a way that the prosthetic leaflet commissure is parallel (anatomical) or perpendicular (anti-anatomical) to the native valve commissure. Usually, surgeons tend to insert bileaflet mitral valves in the antianatomic position in order to increase the likelihood that both leaflets will open symmetrically since diastolic blood flow through the native mitral valve is directed posteriorly. In the anatomic position, the anterior leaflet may not open as readily as the posterior leaflet. However, in the case of tilting-disk valve, the surgeon orients the prosthesis in the mitral position in such a way that the major orifice is directed posteriorly. The rationale being that under physiological conditions, the diastolic flow acquires a laminar pattern and enters the left ventricle through the posterior portion of the mitral inflow tract. Anatomic orientation of a tiltingdisk prosthesis at the mitral position mimics a "trapdoor" effect of the natural anterior mitral leaflet and preserves the left ventricular physiologic vortices during diastole, and hence, pressure gradient generated across the valve is less. ${ }^{10}$ On the contrary, mean prosthetic diastolic gradient will be much higher when the major orifice of a tilting-disk valve would be directed anteriorly rather than posteriorly. ${ }^{11}$ However, the mean transmitral pressure gradient in our patient was $5 \mathrm{~mm} \mathrm{Hg}$, which was reduced to $3 \mathrm{~mm} \mathrm{Hg}$ at the time of discharge.

\section{CONCLUSION}

In conclusion, restriction of mobility of tilting-disk valve at mitral position in the post-CPB period may occur due 
to undue prominence of aortomitral fibrous region following prosthetic aortic valve replacement. Rotating the occluder mechanism of the tilting-disk prosthetic mitral valve may help in overcoming the problem of inadequate disk mobility. Intraoperative TEE is a valuable tool to assess prosthetic valve function in post-CPB period.

\section{REFERENCES}

1. Laub GW, Muralidharan S, Pollock SB, Adkins MS, McGrath LB. The experimental relationship between leaflet clearance and orientation of the St. Jude Medical Valve in the mitral position. J Thorac Cardiovasc Surg 1992 Apr;103(4): 638-641.

2. Machlera H, Perthelb M, Reiterc G, Reiterc U, Zinkd M, Bergmanna P, Waltensdorfer A, Laas J. Influence of bileaflet prosthetic mitral valve orientation on left ventricular flowan experimental in vivo magnetic resonance imaging study. Eur J Cardiothorac Surg 2004 Oct;26(4):747-753.

3. American Society of Anesthesiologists and Society of Cardiovascular Anesthesiologists Task Force on Transesophageal Echocardiography. Practice guidelines for perioperative transesophageal echocardiography. Anesthesiology 2010 May;112(5):1084-1096.

4. Santé P, Renzulli A, Festa M, Vitale N, Mollo A, Dialetto G, De Luca L. Acute postoperative block of mechanical prosthesis. Incidence and treatment. Cardiaovasc Surg 1994 Jun;2(3):403-406.

5. Singh A, Nanda NC, Kirklin JK. Intraoperative transesophageal echocardiographic diagnosis of a stuck bioprosthetic valve leaflet. Echocardiography 2007 Apr;24(4):436-438.

6. Hall KV: Surgical considerations for avoiding disc interference based on a 10-year experience with the Medtronic Hall heart valve. J Card Surg 1988 Jun;3(2):103-108.

7. Talwar S, Jayanthkumar HV, Kumar AS. Chordal preservation during mitral valve replacement: basis, techniques and results. Indian J Thorac Cardiovas 2005 Nov; 21:45-52.

8. Pai GP, Ellison RG, Rubin JW, Moore HV, Kamath MV. Disc immobilization of Bjork-Shiley and Medtronics Hall valves during and immediately after valve replacement. Ann Thorac Surg 1987 Jul;44(1):73-76.

9. Actis Dato GM, Bognolo G, Actis Dato A Jr, Cattaneo C, Di Summa M. A rare complication of mitral valve replacement: sudden cardiac death for immobilization of disc valve by a unraveled suture. J Cardiovasc Surg (Torino) 1995 Apr;36(2):167-169.

10. Akins CW. Long-term results with the Medtronic-Hall valvular prosthesis. Ann Thorac Surg 1996 Mar;61(3):806-813.

11. Pop G, Sutherland GR, Roelandt J, Vletter W, Bos E. What is the ideal orientation of a mitral disc prosthesis? An in vivo haemodynamic study based on color flow imaging and continuous-wave Doppler. Eur Heart J 1989 Apr;10(4): 346-353. 